\title{
Cancer Patients' Utilization of Tertiary Hospitals in Seoul Before and After the Benefit Expansion Policy
}

\author{
Sanghyun Cho', Youngs Chang ${ }^{1}$, Yoon Kim ${ }^{1,2}$ \\ ${ }^{1}$ Department of Health Policy and Management, Seoul National University College of Medicine, Seoul, Korea; ${ }^{2}$ Institute of Health Policy and \\ Management, Seoul National University Medical Research Center, Seoul, Korea
}

Objectives: The aim of this study was to investigate cancer patients' utilization of tertiary hospitals in Seoul before and after the benefit expansion policy implemented in 2013.

Methods: This was a before-and-after study using claims data of the Korean National Health Insurance Service from 2011 to 2016. The unit of analysis was inpatient episodes, and inpatient episodes involving a malignant neoplasm (International Classification of Diseases, Tenth Revision codes: C00-C97) were included in this study. The total sample ( $n=5565$ 076) was divided into incident cases and prevalent cases according to medical use due to cancer in prior years. The tertiary hospitals in Seoul were divided into two groups (the five largest hospitals and the other tertiary hospitals in Seoul).

Results: The proportions of the incident and prevalent episodes occurring in tertiary hospitals in Seoul were 34.9\% and 37.2\%, respectively, of which more than $70 \%$ occurred in the five largest hospitals in Seoul. Utilization of tertiary hospitals in Seoul was higher for inpatient episodes involving cancer surgery, patients with a higher income, patients living in areas close to Seoul, and patients living in areas without a metropolitan city. The utilization of the five largest hospitals increased by 2 percentage points after the policy went into effect.

Conclusions: The utilization of tertiary hospitals in Seoul was concentrated among the five largest hospitals. Future research is necessary to identify the consequences of this utilization pattern.

Key words: Cancer, Choice, Hospital, Health policy

\section{INTRODUCTION}

It has been reported that cancer patients were highly concentrated at tertiary hospitals located in Seoul. In 2009, 49\% of

Received: July 17, 2018 Accepted: January 4, 2019

Corresponding author: Yoon Kim, MD, PhD

Department of Health Policy and Management, Seoul National

University College of Medicine, 103 Daehak-ro, Jongno-gu,

Seoul 03080, Korea

E-mail: yoonkim@snu.ac.kr

This is an Open Access article distributed under the terms of the Creative Commons Attribution Non-Commercial License (http://creativecommons.org/licenses/bync/4.0// which permits unrestricted non-commercial use, distribution, and reproduction in any medium, provided the original work is properly cited. total medical care for cancer patients was performed at medical institutions in Seoul, and 58\% of the income from cancer management at medical institutions in Seoul was accounted for by patients who did not reside in Seoul [1]. Additionally, $58 \%$ of the total medical expenditures of cancer patients occurred at tertiary hospitals, of which $47 \%$ occurred at the 5 largest tertiary hospitals located in Seoul [2].

The benefit expansion policy for 4 major conditions implemented in 2013 was criticized because of the possibility that it would intensify the concentration of cancer patients to tertiary hospitals in Seoul [3]. With the benefit expansion, patients who had previously been limited in their choices due to high expenses would be freer to select the type of medical institu- 
tion where they would receive care, and it was expected that preferences regarding the quality of care would come to play a more important role [4]; therefore, it was likely that patients would be concentrated at tertiary hospitals in Seoul that are expected to provide high-quality care. Furthermore, the benefit expansion would enable cancer patients who had chosen not to receive additional treatment for financial reasons to receive such treatment, leading them to utilize tertiary hospitals in Seoul that provide such treatment [2].

The concentration of cancer patients to tertiary hospitals in Seoul creates various problems. Because the accessibility decreases as the distance between the medical institution and the patient's residential area increases, patients receiving care at a distant hospital cannot receive the required medical services at the appropriate time $[5,6]$. Additionally, an influx of patients exceeding the capacity of a medical institution would increase the waiting time, leading to delays in treatment [7]. Difficulty in access, as well as delays in treatment, adversely affect the health outcomes of cancer patients who require longterm treatment and management [8-11].

Furthermore, unnecessary expenses arise due to repeated tests and the cost and time associated with visits to medical institutions further away from patients' residential areas. The costs resulting from travel expenses and lost time due to cancer treatment were estimated to be 22.9 and 921.1 billion Korean won, respectively [12].

Additionally, the concentration of cancer patients at tertiary hospitals in Seoul can lead to a gap in medical resources among different regions. On one hand, local medical institutions less preferred by patients do not have the capacity to deploy additional medical resources due to financial difficulties [2]. On the other hand, because populations are highly concentrated in the capital region, and patients living in the rural area tend to prefer Seoul over other areas, the tertiary hospitals in Seoul are able to establish large cancer centers and to expand their medical personnel and equipment [13]. While 102 radiation oncologists, accounting for $38 \%$ of all of radiation oncologists nationwide, work in Seoul, there are only 2 radiation oncologists each in Gwangju and Chungbuk, and the number of radiotherapy devices per population was found to be 2 times greater in Seoul than the national average [14]. The lack of medical resources for cancer patients within their region of residence again leads the patients to utilize out-of-region medical institutions.

Previous studies have explored cancer patients' utilization of medical care outside of their residential areas [1,15-17]. However, those studies did not present an in-depth analysis of the concentration of cancer patients at tertiary hospitals in Seoul. Additionally, changes in the utilization of tertiary hospitals in Seoul after the benefit expansion policy, given the criticism of the policy, must be examined using empirical data. The purpose of this study was to investigate cancer patients' utilization of tertiary hospitals in Seoul and to compare its trend before and after the implementation of the benefit expansion policy.

\section{METHODS}

\section{Data and Study Population}

This study used claims data from the Korean National Health Insurance Service. The study population was inpatient episodes involving a malignant neoplasm (International Classification of Diseases, Tenth Revision codes: C00-C97) as the main diagnosis code from 2011 to 2016 [18]. A total of 5565076 cases were selected as the final study population, after excluding 174 cases in which the patient's residential area or the location of the medical institution was unknown and 326775 cases occurred in long-term care hospitals. Among the final study population, cases of the patients who did not have a medical record due to cancer in prior 3 years were classified as incident cases and the other cases were classified as prevalent cases.

\section{Independent Variables}

The episode type, sex, age, income level, residential area, and the type of residential area were used as independent variables, as all these factors may affect cancer patients' choice of medical institution. For episode type, inpatient episodes involving surgical procedures were categorized as surgical episodes, and the others as medical episodes. The patients' income level was divided into quintiles according to the health insurance fee for national health insurance beneficiaries, and Medical Aid patients as a sixth category. Residential area was classified into Seoul, capital regions other than Seoul (Gyeonggi, Incheon), Chungbuk, the Chungnam area (Daejeon, Sejeong, Chungnam), Gangwon, Jeonbuk, the Jeonnam area (Gwangju, Jeonnam), the Gyeongbuk area (Daegu, Gyeongbuk), the Gyeongnam area (Busan, Ulsan, Gyeongnam), and Jeju. The type of residential area was classified as urban (cities having ' $g u$ '), suburban (cities not having 'gu'), and rural ('gun'). 


\section{Dependent Variable}

The dependent variable of this study was whether or not the episode occurred in a tertiary hospital in Seoul. Based on the results of a previous study, which reported that cancer patients were highly concentrated at the 5 largest tertiary hospitals in Seoul [2], the tertiary hospitals in Seoul were divided into (1) the 5 largest tertiary hospitals in Seoul in terms of inpatient volume, and (2) the other tertiary hospitals located in Seoul.

\section{Statistical Analysis}

A before-and-after analysis was conducted to compare the period prior to the benefit expansion policy for 4 major conditions (2011-2013) to the period after the policy (2014-2016). Although the benefit expansion policy went into effect in October 2013, the year 2013 was classified as prior to the policy, because the reduction in out-of-pocket payment by the policy in 2013 was significantly smaller than those in 2014 [19]. The utilization rates of the 5 largest tertiary hospitals in Seoul and the other tertiary hospitals in Seoul according to admission type, income level, residential area, and the type of residential area were calculated for the periods before and after the policy. A logistic regression analysis was conducted to examine the effect of each independent variable, while controlling patients demographic features, on the utilization of 5 largest hospitals in Seoul or on the other tertiary hospitals in Seoul and to identify changes in utilization after the policy.

\section{Ethical Statement}

The study was reviewed and approved by the Institutional Review Board (IRB) of Seoul National University College of Medicine (IRB No. C-1708-035-875). The informed consent was exempted by the board.

\section{RESULTS}

The general characteristics of the study population are presented in Table 1. The number of incident episodes decreased from 1519796 cases to 1398901 cases after the policy. Whereas the number of prevalent episodes slightly increased from 1318895 cases to 1327484 cases after the policy. The proportion of surgical cases was higher in incident episodes than prevalent episodes.

The utilization rate of tertiary hospitals in Seoul before and after the policy for incident episodes is presented in Table 2 . The utilization rate of tertiary hospitals in Seoul increased from
$32.9 \%$ to $34.9 \%$ after the policy. The 5 largest tertiary hospitals accounted for more than $70 \%$ of the utilization of tertiary hospitals in Seoul. While the utilization rate of the 5 largest tertiary hospitals increased from $23.6 \%$ to $28.5 \%$ after the policy, the utilization rate of the other tertiary hospitals in Seoul decreased from $9.3 \%$ to $9.1 \%$. The utilization rate of tertiary hospitals in Seoul was higher for surgical episodes. The increase in the utilization rate after the policy was higher for medical episodes, and this is primarily due to the increase in the utilization of the 5 largest tertiary hospitals. The utilization rate of tertiary hospitals in Seoul in Medical Aid patients was 15.6\%, which was approximately one-third of that of the highest income quintile patients. The utilization rate of tertiary hospitals in Seoul increased in patients of all income levels after the policy, and the magnitude of the change was similar for all income level. Regions with a high utilization of tertiary hospitals in Seoul were Seoul (66.2\%), Chungbuk (40.4\%), the Chungnam area (33.9\%), and Gyeonggi, Incheon (33.4\%), while the regions with a low utilization were the Gyeongnam area $(17.3 \%)$, the Jeonnam area (20.5\%), and the Gyeongbuk area (21.7\%). The utilization rate of tertiary hospitals in Seoul was the highest in urban areas and the lowest in rural areas.

The utilization rate of tertiary hospitals in Seoul before and after the policy for prevalent episodes is presented in Table 3. The utilization rate of tertiary hospitals in Seoul increased from $35.5 \%$ to $37.2 \%$ after the policy. The utilization rates were higher than those of incidents episodes. The utilization rate of the 5 largest tertiary hospitals increased, while the utilization rate of the other tertiary hospitals in Seoul decreased. The utilization rate of tertiary hospitals in Seoul was $20.5 \%$ in Medical Aid patients, and $47.0 \%$ in the highest income quintile patients. The regions with a high utilization of tertiary hospitals in Seoul were Seoul (68.5\%), Chungbuk (44.5\%), Gyeonggi, Incheon (39.0\%), and the Chungnam area (35.9\%), while the regions with a low utilization were the Gyeongnam area (16.9\%), the Jeonnam area (20.6\%), and the Gyeongbuk area (23.6\%). The utilization rate of tertiary hospitals in Seoul was the highest in urban areas and the lowest in rural areas. Similar results were obtained when examined medical and surgical episodes separately; these results are presented in Tables S1 and S2.

The results of the logistic regression analysis are presented in Table 4. Utilization of the 5 largest tertiary hospitals was higher in surgical episodes. There was a more than 4.92 difference in utilization between Medical Aid patients and those in the highest income quintile patients. There was about 4.57 differ- 
Table 1. General characteristics of the study population

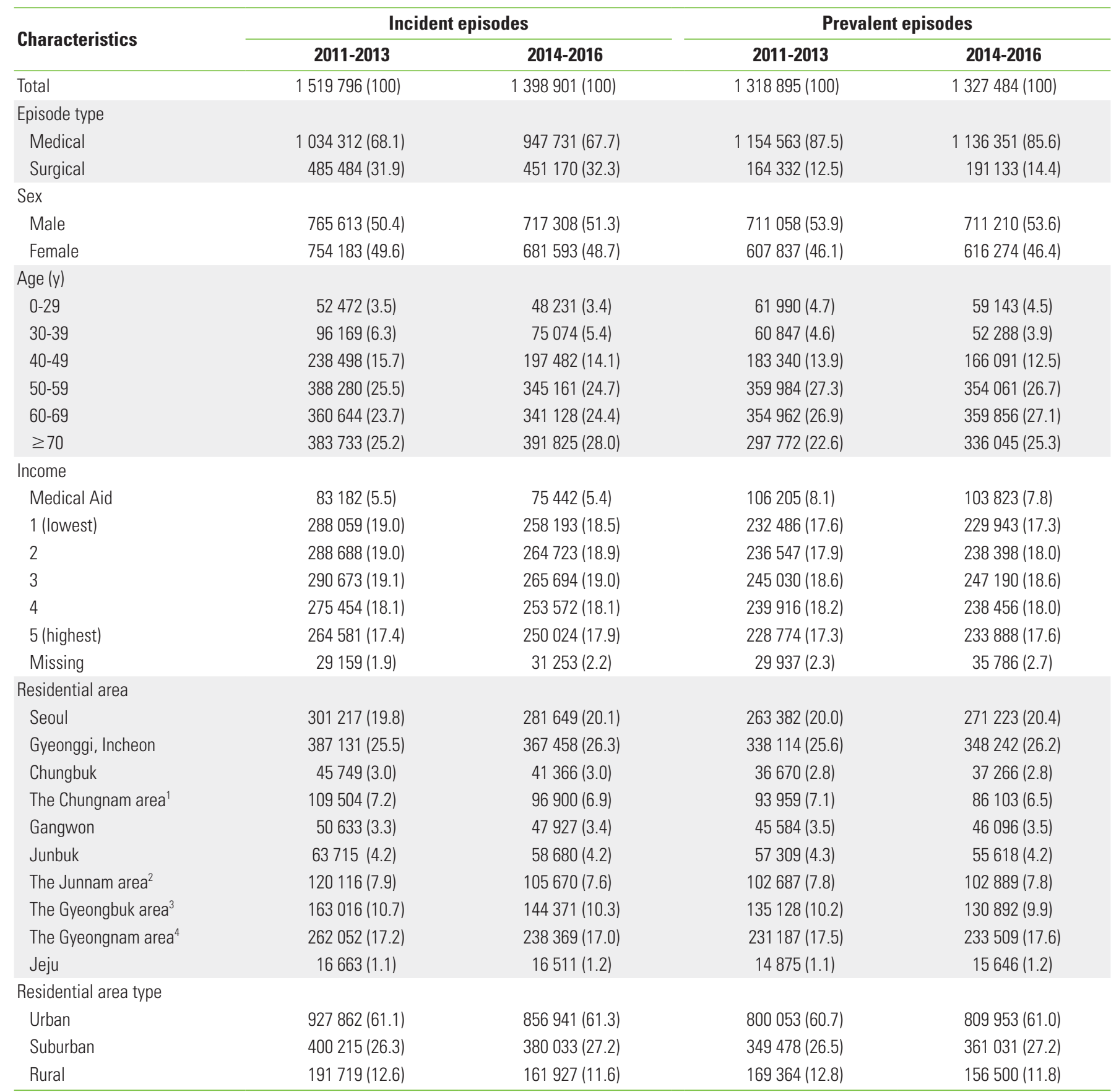

Values are presented as number (\%).

${ }^{1}$ Daejeon, Sejeong, Chungnam.

${ }^{2}$ Gwangju, Jeonnam.

${ }^{3}$ Daegu, Gyeongbuk.

${ }^{4}$ Busan, Ulsan, Gyeongnam.

ence in utilization by residential area, as the Gyeongnam area showed the lowest utilization and Seoul showed the highest. In regions other than Seoul, the utilization was high in Chungbuk, the Chungnam area, and Gyeonggi, Incheon, and low in the Gyeongbuk area and the Jeonnam area. The utilization was lower in urban areas than suburban or rural area. The utilization increased by 1.14 times for incident episodes and by 1.13 times for prevalent episodes after the policy.

For the other tertiary hospitals in Seoul, utilization was higher in medical episodes. The utilization of those hospitals 
Table 2. The proportion of incident episodes occurring at tertiary hospitals in Seoul before and after the benefit expansion policy

\begin{tabular}{|c|c|c|c|c|c|c|c|c|c|}
\hline & \multicolumn{3}{|c|}{ All tertiary hospitals } & \multicolumn{3}{|c|}{ Five largest hospitals } & \multicolumn{3}{|c|}{ The other tertiary hospitals } \\
\hline & Before & After & \multirow{2}{*}{ Diff (\%p) } & Before & After & \multirow{2}{*}{ Diff (\%p) } & Before & After & \multirow{2}{*}{ Diff (\%p) } \\
\hline & 2011-2013 & 2014-2016 & & 2011-2013 & 2014-2016 & & 2011-2013 & 2014-2016 & \\
\hline Total & 32.9 & 34.9 & $2.0^{* * *}$ & 23.6 & 25.8 & $2.2^{* * *}$ & 9.3 & 9.1 & -0.2 \\
\hline \multicolumn{10}{|l|}{ Episode type } \\
\hline Medical & 31.1 & 33.6 & $2.5^{* * *}$ & 21.8 & 24.3 & $2.5^{* * *}$ & 9.2 & 9.2 & 0.0 \\
\hline Surgical & 36.7 & 37.6 & $0.9^{* * *}$ & 27.3 & 28.8 & $1.5^{* * *}$ & 9.4 & 8.7 & $-0.7^{* * *}$ \\
\hline \multicolumn{10}{|l|}{ Income } \\
\hline Medical Aid & 13.7 & 15.6 & $1.9^{* * *}$ & 8.3 & 9.5 & $1.2^{* * *}$ & 5.3 & 6.1 & $0.8^{* * *}$ \\
\hline 1 (lowest) & 28.9 & 31.1 & $2.2^{* * *}$ & 19.5 & 21.9 & $2.4^{* * *}$ & 9.4 & 9.2 & $-0.2^{*}$ \\
\hline 2 & 29.7 & 31.2 & $1.5^{* * *}$ & 20.4 & 22.1 & $1.7^{* * *}$ & 9.2 & 9.1 & -0.2 \\
\hline 3 & 32.8 & 34.7 & $1.9^{* * *}$ & 23.3 & 25.3 & $2.0^{* * *}$ & 9.5 & 9.5 & 0.0 \\
\hline 4 & 37.2 & 39.1 & $1.9^{* * *}$ & 27.4 & 29.7 & $2.3^{* * *}$ & 9.8 & 9.4 & $-0.4^{* * *}$ \\
\hline 5 (highest) & 42.3 & 44.2 & $1.9^{* * *}$ & 32.6 & 35.1 & $2.5^{* * *}$ & 9.7 & 9.1 & $-0.6^{* * *}$ \\
\hline Missing & 32.6 & 36.1 & $3.5^{* * *}$ & 24.3 & 27.7 & $3.4^{* * *}$ & 8.3 & 8.4 & 0.1 \\
\hline \multicolumn{10}{|l|}{ Residential area } \\
\hline Seoul & 65.3 & 66.2 & $0.9^{* * *}$ & 39.6 & 41.2 & $1.6^{* * *}$ & 25.6 & 25.0 & $-0.6^{* * *}$ \\
\hline Gyeonggi, Incheon & 32.4 & 33.4 & $1.0^{* * *}$ & 23.5 & 25.2 & $1.7^{* * *}$ & 8.9 & 8.2 & $-0.7^{* * *}$ \\
\hline Chungbuk & 37.0 & 40.4 & $3.4^{* * *}$ & 30.2 & 33.6 & $3.4^{* * *}$ & 6.9 & 6.8 & -0.1 \\
\hline The Chungnam area ${ }^{1}$ & 29.0 & 33.9 & $4.9^{* * *}$ & 23.8 & 28.5 & $4.7^{* * *}$ & 5.3 & 5.5 & $0.2^{*}$ \\
\hline Gangwon & 29.3 & 32.0 & $2.7^{* * *}$ & 23.9 & 26.4 & $2.5^{* * *}$ & 5.5 & 5.6 & 0.1 \\
\hline Junbuk & 20.6 & 23.4 & $2.8^{* * *}$ & 16.9 & 20.1 & $3.2^{* * *}$ & 3.7 & 3.3 & $-0.3^{* * *}$ \\
\hline The Junnam area $^{2}$ & 18.3 & 20.5 & $2.2^{* * *}$ & 15.0 & 17.4 & $2.4^{* * *}$ & 3.3 & 3.0 & $-0.3^{* * *}$ \\
\hline The Gyeongbuk area ${ }^{3}$ & 19.8 & 21.7 & $1.9^{* * *}$ & 16.8 & 18.7 & $1.9^{* * *}$ & 3.0 & 3.1 & 0.1 \\
\hline The Gyeongnam area ${ }^{4}$ & 15.9 & 17.3 & $1.4^{* * *}$ & 13.7 & 15.1 & $1.4^{* * *}$ & 2.2 & 2.1 & $0.0^{*}$ \\
\hline Jeju & 29.7 & 33.9 & $4.2^{* * *}$ & 24.7 & 29.0 & $4.3^{* * *}$ & 5.0 & 4.9 & -0.2 \\
\hline \multicolumn{10}{|l|}{ Residential area type } \\
\hline Urban & 35.8 & 37.8 & $2.0^{* * *}$ & 24.7 & 26.9 & $2.2^{* * *}$ & 11.1 & 10.9 & $-0.2^{* * *}$ \\
\hline Suburban & 30.3 & 32.3 & $2.0^{* * *}$ & 23.2 & 25.4 & $2.2^{* * *}$ & 7.1 & 6.8 & $-0.3^{* * *}$ \\
\hline Rural & 24.2 & 25.5 & $1.3^{* * *}$ & 19.1 & 20.9 & $1.8^{* * *}$ & 5.1 & 4.6 & $-0.5^{* * *}$ \\
\hline
\end{tabular}

Values are presented as \%.

Diff, difference.

'Daejeon, Sejeong, Chungnam.

${ }^{2}$ Gwangju, Jeonnam.

${ }^{3}$ Daegu, Gyeongbuk.

${ }^{4}$ Busan, Ulsan, Gyeongnam.

${ }^{*} p<0.05,{ }^{* *} p<0.001$.

was the highest in Seoul which was 18-fold higher than the lowest region (the Gyeongnam area). The utilization decreased for both incident episodes and prevalent episodes after the policy. Similar results were observed when examined medical and surgical episodes separately; these results are presented in Tables S3 and S4.

\section{DISCUSSION}

This study investigated the current status of cancer patients' utilization of tertiary hospitals in Seoul and compared its trends before and after the implementation of the benefit expansion policy for 4 major conditions. A previous study that analyzed cancer patients' medical utilization from 1999 to 2005 using the cancer registration data and claims data reported that the utilization rate of the 4 largest tertiary hospitals in Seoul was 19.8\%, while the utilization rate of the other tertiary hospitals in Seoul was $17.3 \%$ [17]. In this study, the utilization rates of the 5 largest tertiary hospitals in Seoul from 2014 to 2016 for the incident and prevalent episodes were $25.8 \%, 28.3 \%$, respectively while the utilization rates of the other tertiary hospitals in Seoul were 9.1\%, 8.9\%, respectively. 
Table 3. The proportion of prevalent episodes occurring at tertiary hospitals in Seoul before and after the benefit expansion policy

\begin{tabular}{|c|c|c|c|c|c|c|c|c|c|}
\hline & \multicolumn{3}{|c|}{ All tertiary hospitals } & \multicolumn{3}{|c|}{ Five largest hospitals } & \multicolumn{3}{|c|}{ The other tertiary hospitals } \\
\hline & Before & After & \multirow{2}{*}{ Diff (\%p) } & Before & After & \multirow{2}{*}{ Diff (\%p) } & Before & After & \multirow{2}{*}{ Diff (\%p) } \\
\hline & 2011-2013 & 2014-2016 & & 2011-2013 & 2014-2016 & & 2011-2013 & 2014-2016 & \\
\hline Total & 35.5 & 37.2 & $1.7^{* * *}$ & 26.1 & 28.3 & $2.1^{* * *}$ & 9.3 & 8.9 & $-0.4^{* * *}$ \\
\hline \multicolumn{10}{|l|}{ Episode type } \\
\hline Medical & 33.9 & 35.7 & $1.8^{* * *}$ & 24.4 & 26.6 & $2.2^{* * *}$ & 9.5 & 9.1 & $-0.4^{* * *}$ \\
\hline Surgical & 46.5 & 46.3 & -0.2 & 38.4 & 38.5 & 0.1 & 8.1 & 7.7 & $-0.4^{* * *}$ \\
\hline \multicolumn{10}{|l|}{ Income } \\
\hline Medical Aid & 17.8 & 20.5 & $2.7^{* * *}$ & 11.2 & 13.5 & $2.3^{* * *}$ & 6.6 & 7.0 & $0.4^{*}$ \\
\hline 1 (lowest) & 32.4 & 33.8 & $1.4^{* * *}$ & 22.5 & 24.9 & $2.4^{* * *}$ & 9.9 & 9.0 & $-0.9^{* * *}$ \\
\hline 2 & 32.6 & 34.1 & $1.5^{* * *}$ & 22.8 & 24.5 & $1.7^{* * *}$ & 9.8 & 9.6 & $-0.2^{*}$ \\
\hline 3 & 35.4 & 37.5 & $2.1^{* * *}$ & 25.8 & 28.2 & $2.4^{* * *}$ & 9.6 & 9.3 & $-0.3^{*}$ \\
\hline 4 & 39.6 & 40.6 & $1.0^{* * *}$ & 30.1 & 31.5 & $1.4^{* * *}$ & 9.5 & 9.1 & $-0.4^{* * *}$ \\
\hline 5 (highest) & 45.6 & 47.0 & $1.4^{* * *}$ & 36.5 & 38.5 & $2.0^{* * *}$ & 9.2 & 8.5 & $-0.7^{* * *}$ \\
\hline Missing & 35.3 & 39.7 & $4.4^{* * *}$ & 25.8 & 30.8 & $5.0^{* * *}$ & 9.5 & 8.9 & $-0.6^{*}$ \\
\hline \multicolumn{10}{|l|}{ Residential area } \\
\hline Seoul & 68.5 & 68.5 & 0.1 & 43.5 & 45.1 & $1.6^{* * *}$ & 24.9 & 23.4 & $-1.5^{* * *}$ \\
\hline Gyeonggi, Incheon & 38.4 & 39.0 & $0.6^{* * *}$ & 28.9 & 30.4 & $1.5^{* * *}$ & 9.5 & 8.6 & $-0.9^{* * *}$ \\
\hline Chungbuk & 38.4 & 44.5 & $6.1^{* * *}$ & 30.5 & 37.5 & $7.0^{* * *}$ & 7.9 & 6.9 & $-1.0^{* * *}$ \\
\hline The Chungnam area ${ }^{1}$ & 31.6 & 35.9 & $4.3^{* * *}$ & 26.0 & 30.5 & $4.5^{* * *}$ & 5.5 & 5.5 & 0.0 \\
\hline Gangwon & 29.9 & 32.4 & $2.5^{* * *}$ & 24.8 & 27.0 & $2.2^{* * *}$ & 5.0 & 5.4 & $0.4^{*}$ \\
\hline Junbuk & 22.0 & 24.5 & $2.5^{* * *}$ & 18.4 & 20.8 & $2.4^{* * *}$ & 3.6 & 3.7 & 0.1 \\
\hline The Junnam area² & 18.8 & 20.6 & $1.8^{* * *}$ & 15.6 & 17.3 & $1.7^{* * *}$ & 3.2 & 3.3 & 0.1 \\
\hline The Gyeongbuk area ${ }^{3}$ & 20.9 & 23.6 & $2.7^{* * *}$ & 17.6 & 20.3 & $2.7^{* * *}$ & 3.3 & 3.3 & 0.0 \\
\hline The Gyeongnam area ${ }^{4}$ & 15.7 & 16.9 & $1.2^{* * *}$ & 13.6 & 14.7 & $1.1^{* * *}$ & 2.0 & 2.1 & $0.1^{*}$ \\
\hline Jeju & 28.7 & 31.7 & $3.0^{* * *}$ & 24.5 & 27.1 & $2.6^{* * *}$ & 4.2 & 4.6 & 0.4 \\
\hline \multicolumn{10}{|l|}{ Residential area type } \\
\hline Urban & 38.7 & 40.2 & $1.5^{* * *}$ & 27.7 & 29.7 & $2.0^{* * *}$ & 11.0 & 10.5 & $-0.5^{* * *}$ \\
\hline Suburban & 33.0 & 34.8 & $1.8^{* * *}$ & 25.4 & 27.8 & $2.4^{* * *}$ & 7.6 & 7.0 & $-0.6^{* * *}$ \\
\hline Rural & 25.7 & 27.2 & $1.5^{* * *}$ & 20.5 & 22.3 & $1.8^{* * *}$ & 5.2 & 5.0 & $-0.2^{*}$ \\
\hline
\end{tabular}

Values are presented as \%.

Diff, difference.

${ }^{1}$ Daejeon, Sejeong, Chungnam.

${ }^{2}$ Gwangju, Jeonnam.

${ }^{3}$ Daegu, Gyeongbuk.

${ }^{4}$ Busan, Ulsan, Gyeongnam.

${ }^{*} p<0.05,{ }^{* * *} p<0.001$.

The logistic regression analysis showed that the utilization of the 5 largest tertiary hospitals in Seoul increased after the policy, whereas the utilization of the other tertiary hospitals in Seoul decreased. These results indicate that cancer patients are concentrated to a few largest hospitals in Seoul, and this trend is intensifying over time.

Regional differences in cancer patients' utilization of the 5 largest tertiary hospitals in Seoul can be explained by the differences in accessibility to Seoul between regions. Cancer patients choose medical institutions primarily by their size, repu- tation, and new technologies $[20,21]$. Accessibility acts as one of the constraints for cancer patients to choose the medical institution; choices become more limited as the distance to the medical institution increases [22]. This can explain the high utilization of the 5 largest tertiary hospitals in Seoul among the patients living in the Chungnam area, Chungbuk, and Gyeonggi, Incheon, as well as the greater increase in the utilization after the policy in the Chungnam area and Chungbuk. Other than those regions, regions that showed high utilization of the 5 largest tertiary hospitals in Seoul and a large increase 
Table 4. Multiple logistic regression analysis of utilization of the 5 largest hospitals and the other tertiary hospitals in Seoul

\begin{tabular}{|c|c|c|c|c|}
\hline & \multicolumn{2}{|c|}{ Five largest hospitals } & \multicolumn{2}{|c|}{ The other tertiary hospitals } \\
\hline & Incident episodes & Prevalent episodes & Incident episodes & Prevalent episodes \\
\hline \multicolumn{5}{|l|}{ Episode type } \\
\hline Medical & 1.00 (reference) & 1.00 (reference) & 1.00 (reference) & 1.00 (reference) \\
\hline Surgical & $1.31(1.30,1.32)$ & $2.01(1.99,2.02)$ & $0.94(0.93,0.95)$ & $0.81(0.80,0.82)$ \\
\hline \multicolumn{5}{|l|}{ Sex } \\
\hline Male & $1.27(1.26,1.28)$ & $1.16(1.15,1.16)$ & $0.83(0.82,0.84)$ & $0.85(0.85,0.86)$ \\
\hline Female & 1.00 (reference) & 1.00 (reference) & 1.00 (reference) & 1.00 (reference) \\
\hline \multicolumn{5}{|l|}{ Age (y) } \\
\hline $0-29$ & $5.48(5.40,5.56)$ & $7.18(7.08,7.28)$ & $0.77(0.75,0.79)$ & $0.55(0.54,0.57)$ \\
\hline $30-39$ & $2.11(2.08,2.14)$ & $2.34(2.30,2.37)$ & $1.42(1.40,1.45)$ & $1.11(1.08,1.13)$ \\
\hline $40-49$ & $1.77(1.75,1.79)$ & $1.83(1.81,1.84)$ & $1.31(1.29,1.33)$ & $1.15(1.13,1.17)$ \\
\hline $50-59$ & $1.79(1.78,1.81)$ & $1.77(1.76,1.79)$ & $1.22(1.20,1.23)$ & $1.16(1.15,1.17)$ \\
\hline $60-69$ & $1.66(1.65,1.68)$ & $1.60(1.58,1.61)$ & $1.17(1.16,1.19)$ & $1.16(1.14,1.17)$ \\
\hline$\geq 70$ & 1.00 (reference) & 1.00 (reference) & 1.00 (reference) & 1.00 (reference) \\
\hline \multicolumn{5}{|l|}{ Income } \\
\hline Medical Aid & 1.00 (reference) & 1.00 (reference) & 1.00 (reference) & 1.00 (reference) \\
\hline 1 (lowest) & $2.32(2.28,2.37)$ & $2.13(2.09,2.16)$ & $1.47(1.44,1.51)$ & $1.31(1.28,1.34)$ \\
\hline 2 & $2.45(2.40,2.49)$ & $2.14(2.10,2.17)$ & $1.46(1.43,1.50)$ & $1.36(1.33,1.39)$ \\
\hline 3 & $2.91(2.86,2.96)$ & $2.59(2.55,2.63)$ & $1.50(1.47,1.54)$ & $1.33(1.30,1.36)$ \\
\hline 4 & $3.65(3.59,3.72)$ & $3.18(3.13,3.23)$ & $1.55(1.52,1.59)$ & $1.29(1.26,1.32)$ \\
\hline 5 (highest) & $4.92(4.83,5.01)$ & $4.42(4.36,4.49)$ & $1.57(1.54,1.61)$ & $1.23(1.21,1.26)$ \\
\hline Missing & $3.13(3.05,3.22)$ & $2.69(2.63,2.75)$ & $1.36(1.31,1.42)$ & $1.32(1.28,1.36)$ \\
\hline \multicolumn{5}{|l|}{ Residential area } \\
\hline Seoul & $4.57(4.53,4.62)$ & $5.59(5.53,5.65)$ & $18.77(18.38,19.16)$ & $18.47(18.07,18.88)$ \\
\hline Gyeonggi, Incheon & $1.78(1.77,1.80)$ & $2.40(2.38,2.43)$ & $3.95(3.87,4.04)$ & $4.37(4.27,4.47)$ \\
\hline Chungbuk & $2.70(2.65,2.74)$ & $3.05(3.00,3.11)$ & $2.91(2.81,3.01)$ & $3.22(3.11,3.33)$ \\
\hline The Chungnam area' & $2.03(2.00,2.06)$ & $2.30(2.27,2.34)$ & $2.39(2.33,2.46)$ & $2.53(2.46,2.60)$ \\
\hline Gangwon & $1.77(1.74,1.80)$ & $1.91(1.87,1.94)$ & $1.97(1.90,2.04)$ & $1.86(1.79,1.93)$ \\
\hline Junbuk & $1.26(1.24,1.29)$ & $1.42(1.40,1.45)$ & $1.39(1.34,1.44)$ & $1.48(1.42,1.54)$ \\
\hline The Junnam area ${ }^{2}$ & $1.09(1.07,1.10)$ & $1.16(1.14,1.18)$ & $1.28(1.25,1.32)$ & $1.36(1.32,1.40)$ \\
\hline The Gyeongbuk area ${ }^{3}$ & $1.23(1.21,1.24)$ & $1.35(1.33,1.37)$ & $1.31(1.28,1.35)$ & $1.47(1.43,1.51)$ \\
\hline The Gyeongnam area ${ }^{4}$ & 1.00 (reference) & 1.00 (reference) & 1.00 (reference) & 1.00 (reference) \\
\hline Jeju & $1.77(1.73,1.82)$ & $1.73(1.68,1.78)$ & $1.71(1.62,1.80)$ & $1.55(1.46,1.65)$ \\
\hline \multicolumn{5}{|l|}{ Residential area type } \\
\hline Urban & $0.79(0.79,0.80)$ & $0.80(0.80,0.81)$ & $0.59(0.58,0.60)$ & $0.60(0.59,0.61)$ \\
\hline Suburban & $1.09(1.08,1.10)$ & $1.07(1.05,1.08)$ & $1.02(1.00,1.04)$ & $1.04(1.02,1.06)$ \\
\hline Rural & 1.00 (reference) & 1.00 (reference) & 1.00 (reference) & 1.00 (reference) \\
\hline \multicolumn{5}{|l|}{ Time period } \\
\hline Before policy & 1.00 (reference) & 1.00 (reference) & 1.00 (reference) & 1.00 (reference) \\
\hline After policy & $1.14(1.14,1.15)$ & $1.13(1.12,1.13)$ & $0.97(0.96,0.98)$ & $0.94(0.93,0.95)$ \\
\hline
\end{tabular}

Values are presented as odds ratio ( $95 \%$ confidence interval).

'Daejeon, Sejeong, Chungnam.

${ }^{2}$ Gwangju, Jeonnam.

${ }^{3}$ Daegu, Gyeongbuk.

${ }^{4}$ Busan, Ulsan, Gyeongnam.

in utilization after the policy were regions without metropolitan cities, such as Gangwon, Jeju, and Jeonbuk. These results can be interpreted by the regional gaps in medical resources.
Eleven of the 15 tertiary hospitals in Korea with more than 1000 beds are located in metropolitan cities, and local areas have fewer types and numbers of medical equipment than 
metropolitan cities [23]. The low utilization of the 5 largest tertiary hospitals in Seoul among patients residing in urban areas in the logistic regression analysis supports this interpretation.

The gap in the utilization of the 5 largest tertiary hospitals in Seoul between Medical Aid patients and the highest income quintile patients was more than 4-fold which is consistent with the results of previous studies $[16,17,24]$. There were no significant differences between the income levels in the increase in the utilization after the benefit expansion policy. Unequal accessibility to medical services may lead to unequal health outcome in cancer patients. A previous study which investigated survival rates of cancer patients by income level revealed that the 1-year, 3-year, and 5-year survival rates were all higher in patients with higher income [24].

After the introduction of the benefit expansion policy for 4 major conditions, the utilization rate of the 5 largest tertiary hospitals in Seoul increased for both incident episodes and prevalent episodes, and the increase was higher for medical episodes than for surgical episodes. If this increase was due to the benefit expansion policy, it can be interpreted that for surgery, which is a relatively high-risk medical service, the preference for the quality of care was evident [25] so that the cost change did not significantly affect the choice of the medical institution in cancer patients, whereas for non-surgical services, the cost played a relatively important role in the selection of the medical institution. Meanwhile, contrary to the results of previous studies, which reported that the increase in medical use after the benefit expansion policy was higher among lower-income patients [26-28], the gap between income levels in the utilization of tertiary hospitals in Seoul did not change after the policy in this study. This discrepancy may have been due to differences in the outcome variable measured between this study and prior studies. The outcome variable in this study, the utilization of tertiary hospitals in Seoul, has a geographic feature. Patients who reside in rural areas must spend a large amount of time both traveling to tertiary hospitals in Seoul and waiting to receive care at those hospitals. It may be that the access barrier prevents lower-income patients to respond to the policy, given that time available for medical use is generally low in the lower-income patients.

There are some limitations to this study. Various operational definitions are in use to define the medical use of cancer patients using medical claims data. Although this study used the operational definition known to have the highest validity, it remains possible that the use of medical services may have been overestimated in some cases [18]. Additionally, the severity of cancer, which may significantly affect patients' choice of medical institutions, was not included in this analysis. Using cancer registration data may overcome these limitations in future studies.

This was a descriptive study that examined the current status of the concentration of cancer patients at tertiary hospitals in Seoul. Future studies are necessary to investigate the different aspects and the consequences of this utilization pattern. Various other indicators, such as the number of patients or medical expenses, can be used to analyze the utilization pattern of medical institutions, which may produce different results [29]. Researches using various indicators to capture different aspects of the cancer patients' utilization pattern will broaden the understanding of this phenomenon. In addition, it is necessary to identify 'appropriate' patient concentration by analyzing the concentration pattern by cancer type and its stage. For highrisk surgical procedures and rare cancers, the concentration of cancer patients at a few medical institutions can increase the quality of care [30-32], and a greater concentration of such cases can be considered 'appropriate'. For the other cases, quantitative research is needed to assess the effect of the patient concentration on patient health and social cost. Such studies will provide a basis for the establishment of a regional cancer management system and for the provision of integrated cancer care currently implemented by the government.

\section{SUPPLEMENTARY MATERIALS}

Supplementary materials are available at https://www.jpmph. org/.

\section{CONFLICT OF INTEREST}

The authors have no conflicts of interest associated with the material presented in this paper.

\section{ACKNOWLEDGEMENTS}

This article was based on a thesis submitted by the first author to Seoul National University College of Medicine in partial fulfillment for the Master of Science in Medicine.

This research was supported by the Korean National Health Insurance Service Atlas Project funded by the Korean National Health Insurance Service (NHIS-2017-1-309). 


\section{ORCID}

Sanghyun Cho https://orcid.org/0000-0002-2081-1076

Youngs Chang https://orcid.org/0000-0003-4722-6894

Yoon Kim https://orcid.org/0000-0001-7257-1679

\section{REFERENCES}

1. Health Insurance Policy Research Institute. Analysis of medical utilization pattern by region using health insurance claims data: focusing on cancer [cited 2018 Dec 15]. Available from: http:// lib.nhis.or.kr/search/detail/CATXAZ000000002560 (Korean).

2. Korea Institute for Health and Social Affair. Improving the sustainability of healthcare delivery in line with $\mathrm{NHI}$ coverage enhancement [cited 2018 Dec 15]. Available from: https://www. kihasa.re.kr/common/filedown.do?seq=16546 (Korean).

3. Kang HJ. Policy direction for decreasing the concentration of patients to extra-large hospitals. Health Welf Policy Forum 2014;(210):65-76 (Korean).

4. Dranove D, Satterthwaite MA. Monopolistic competition when price and quality are imperfectly observable. RAND J Econ 1992; 23(4):518-534.

5. Mooney C, Zwanziger J, Phibbs CS, Schmitt S. Is travel distance a barrier to veterans' use of VA hospitals for medical surgical care? Soc Sci Med 2000;50(12):1743-1755.

6. Nemet GF, Bailey AJ. Distance and health care utilization among the rural elderly. Soc Sci Med 2000;50(9):1197-1208.

7. Siciliani L, Hurst J. Tackling excessive waiting times for elective surgery: a comparative analysis of policies in 12 OECD countries. Health Policy 2005;72(2):201-215.

8. King M, Jones L, Richardson A, Murad S, Irving A, Aslett $H$, et al. The relationship between patients' experiences of continuity of cancer care and health outcomes: a mixed methods study. Br J Cancer 2008;98(3):529-536.

9. Shin DW, Cho J, Kim SY, Guallar E, Hwang SS, Cho B, et al. Delay to curative surgery greater than 12 weeks is associated with increased mortality in patients with colorectal and breast cancer but not lung or thyroid cancer. Ann Surg Oncol 2013; 20(8):2468-2476.

10. Yun YH, Kim YA, Min YH, Park S, Won YJ, Kim DY, et al. The influence of hospital volume and surgical treatment delay on long-term survival after cancer surgery. Ann Oncol 2012;23(10): 2731-2737.

11. Massarweh NN, Chiang YJ, Xing Y, Chang GJ, Haynes AB, You $\mathrm{YN}$, et al. Association between travel distance and metastatic disease at diagnosis among patients with colon cancer. J Clin Oncol 2014;32(9):942-948.

12. Health Insurance Policy Research Institute. Analysis of the social costs of major diseases for setting health care policy priorities [cited 2018 Dec 10]. Available from: https://www.dbpia. co.kr/SKnowledge/ArticleDetail/NODE06281903 (Korean).

13. Kim KS. Polarization of cancer patient management. J Korean Med Assoc 2017;60(3):223-227 (Korean).

14. Park SK, Kim JE, Lee HJ, Kim YS, Lee YJ, Kim SM, et al. Nationwide survey on health and medical service. Cheongju: Korea Health Industry Development Institute; 2018 (Korean).

15. Moon YO, Park EC, Shin HR, Won YJ, Jung KW, Lee JH, et al. Regional variation in accessing regional hospitals for cancer patients. Korean J Epidemiol 2006;28(2):152-161 (Korean).

16. Park JH, Park EC, Ham MI, Park JH, Lee HY, Won YJ, et al. Study on inequalities in relation to accessibility of cancer patients by regions. Goyang: National Cancer Center; 2009, p. 16-43 (Korean).

17. Ministry of Health and Welfare. Inequality on medical service utilization among cancer patients across income groups [cited 2018 Dec 15]. Available from: http://www.mohw.go.kr/react/al/ sal0301vw.jsp?PAR_MENU_ID=04\&MENU_ID=0403\&page = 653\&CONT_SEQ $=38789$ (Korean).

18. National Health Insurance Service Ilsan Hospital Research Center. Study on the operational definition of diseases using health insurance claims data [cited 2018 Dec 20]. Available from: http://www.alio.go.kr/informationResearchView.do? seq $=2341987$ (Korean).

19. Health Insurance Review and Assessment Service. Study on the effect of benefit expansion policy [cited 2018 Dec 20]. Available from: http://www.alio.go.kr/informationResearchView. do?seq=2384373 (Korean).

20. Youn Kl, Doh SR. An analysis of the diseases specific medical service organization selection factors of patients. Korea J Hosp Manag 2007;12(4):1-21 (Korean).

21. Chang DM. A study on utilization behavior of cancer patients. Korean J Public Health 1998;24(2):106-117 (Korean).

22. Smith KB, Humphreys JS, Wilson MG. Addressing the health disadvantage of rural populations: how does epidemiological evidence inform rural health policies and research? Aust J Rural Health 2008;16(2):56-66.

23. National Cancer Center. A study on mid-term assessment of designation and operational performance of the regional cancer centers in Korea [cited 2018 Dec 10]. Available from: http://www.ndsl.kr/ndsl/search/detail/report/reportSearch 
ResultDetail.do?cn =TRKO201700009041 (Korean).

24. Yoon TH, Lee SY, Kim CW, Kim SY, Jeong BG, Park HK. Inequalities in medical care utilization by South Korean cancer patients according to income: a retrospective cohort study. Int J Health Serv 2011;41(1):51-66.

25. Varkevisser M, van der Geest SA. Why do patients bypass the nearest hospital? An empirical analysis for orthopaedic care and neurosurgery in the Netherlands. Eur J Health Econ 2007; 8(3):287-295.

26. Kim SJ, Ko Y, Oh JH, Kwon SM. The effect of expanding health insurance benefits for cancer patients on the equity in health care utilization. Health Policy Manag 2008;18(3):90-109 (Korean).

27. Bae JY. Impacts of health insurance coverage expansion on health care utilization and health status. Korean J Soc Welf Stud 2010;41(2):35-65 (Korean).
28. Hong J. The effects of the health insurance coverage expansion for cancer on health care utilization and health outcomes. Korea Rev Appl Econ 2016;18(4):5-42 (Korean).

29. Kim JH, Lee JH, Lee JH. Changes in healthcare utilizations of cancer patients since the launch of KTX. J Korean Soc Railway 2010;13(2):236-243 (Korean).

30. Begg CB, Cramer LD, Hoskins WJ, Brennan MF. Impact of hospital volume on operative mortality for major cancer surgery. JAMA 1998;280(20):1747-1751.

31. Birkmeyer JD, Siewers AE, Finlayson EV, Stukel TA, Lucas FL, Batista I, et al. Hospital volume and surgical mortality in the United States. N Engl J Med 2002;346(15):1128-1137.

32. Shin DW, Cho J, Yang HK, Kim SY, Lee SH, Suh B, et al. Oncologist perspectives on rare cancer care: a nationwide survey. Cancer Res Treat 2015;47(4):591-599. 